\title{
Echinococcal tension pneumothorax
}

\author{
FARHAN BAKIR AND MUAYYAD M. AL-OMERI \\ From the Medical Department and Cardio-thoracic Unit, Medical College and Republic Hospital, \\ University of Baghdad, Baghdad, Iraq
}

Hydatid cyst is rarely mentioned among the causes of pneumothorax in text-books or monographs, especially those written in English. Five examples of tension pneumothorax secondary to ruptured hydatid cyst of the lung are reported: the mechanism of this tension effect and helpful diagnostic points are discussed. We think that surgical correction is the only satisfactory treatment of tension pneumothorax due to ruptured hydatid cyst: surgery is advocated in any suspected cyst as soon as it is discovered so as to avoid any such serious complication.

Hydatid cyst is rarely listed among the aetiological causes of spontaneous pneumothorax in the English literature (Perry, 1939 ; DuBose, Price, and Guilfoil, 1953 ; Mills and Baisch, 1965).

References to echinococcus as a cause of pneumothorax are scanty even from countries in which the disease is known to be prevalent or endemic (Sinclair, 1955). Among 478 cases of pulmonary hydatid cyst seen in Australia and New Zealand, hydropneumothorax occurred in $2.4 \%$ of the 246 cases from Australia but was not reported among the 232 New Zealand cases (Waddle, 1950). In a previous study on pulmonary hydatid cyst in Iraq only one case of pneumothorax was listed among the 189 cases reported (Kelly and Izzi, 1959).

However, we found some references in the Spanish medical literature to pneumothorax complicating pulmonary hydatids (Sala de Pablo and San Joaquin, 1954). Tomalino (1959), in his paper on pleural complications, gave 16 examples of pneumothorax, with mediastinal shift in five cases. Agustoni, Taboada, Berinstein, and Chorne (1960) have reported a single case of tension pneumothorax.

The purpose of this paper is to present five cases of tension pneumothorax due to pulmonary hydatid cysts. All the five patients were seen within a period of one year.

\section{CASE REPORTS}

CASE 1 A 12-year-old boy complained of sudden shortness of breath which was preceded by right-sided pleuritic pain, stabbing in nature, together with a dry irritative cough. Physical examination revealed a seriously sick child with severe respiratory distress and orthopnoca. Respiration was shallow and the rate was 42 per minute; the pulse rate was 116 per minute and the temperature $38^{\circ} \mathrm{C}$. The trachea was well shifted to the left and the apex beat was pushed to the mid-axillary line. The classical signs of fluid were demonstrated in the lower part of the right hemithorax, and signs of air were found in the upper part of the right pleural cavity. A chest radiograph confirmed the presence of hydropneumothorax (Fig. 1a). The W.B.C. ranged between 9,000 and $10,000 / \mathrm{cu}$. $\mathrm{mm}$. There were $76 \%$ polymorphs and $6 \%$ eosinophils. On aspiration of the chest, air under high pressure gushed out, and was followed by turbid fluid. A repeat chest radiograph failed to show improvement, so a watersealed drainage tube was inserted, and suction applied. The symptoms improved, the trachea became central, and a further radiograph showed correction of the mediastinal shift, though the lung was still collapsed. There was an ill-defined shadow at the right cardiophrenic angle with an irregular thin wall border (Fig. 1b). The Casoni skin test was positive. Because of the failure of conservative measures a thoracotomy was performed, when a huge ruptured hydatid cyst was found destroying and replacing the middle lobe, which was therefore removed. Lung expansion was complete and recovery was satisfactory (Fig. 1c).

CASE 2 A 26-year-old girl had been in normal health until four days prior to admission, when she fell down stairs on her right side. She developed severe distressing pleural pain with irritative cough and haemoptysis. The pain increased on coughing and breathing. After the chest had been strapped for a suspected fractured rib, rigor, fever, and progressive shortness of breath developed.

Physical examination revealed a sick woman who was in pain and had severe dyspnoea. The respiratory rate was $38 /$ minute, the pulse rate $110 /$ minute, the temperature $39^{\circ} \mathrm{C}$, and the trachea was pushed to the left. Signs of hydropneumothorax were elicited in the right hemithorax, and the liver was enlarged to about $3 \mathrm{~cm}$. below the costal margin. Her chest radiograph demonstrated a partial collapse of the right lung with air at the top and fluid at the bottom of 


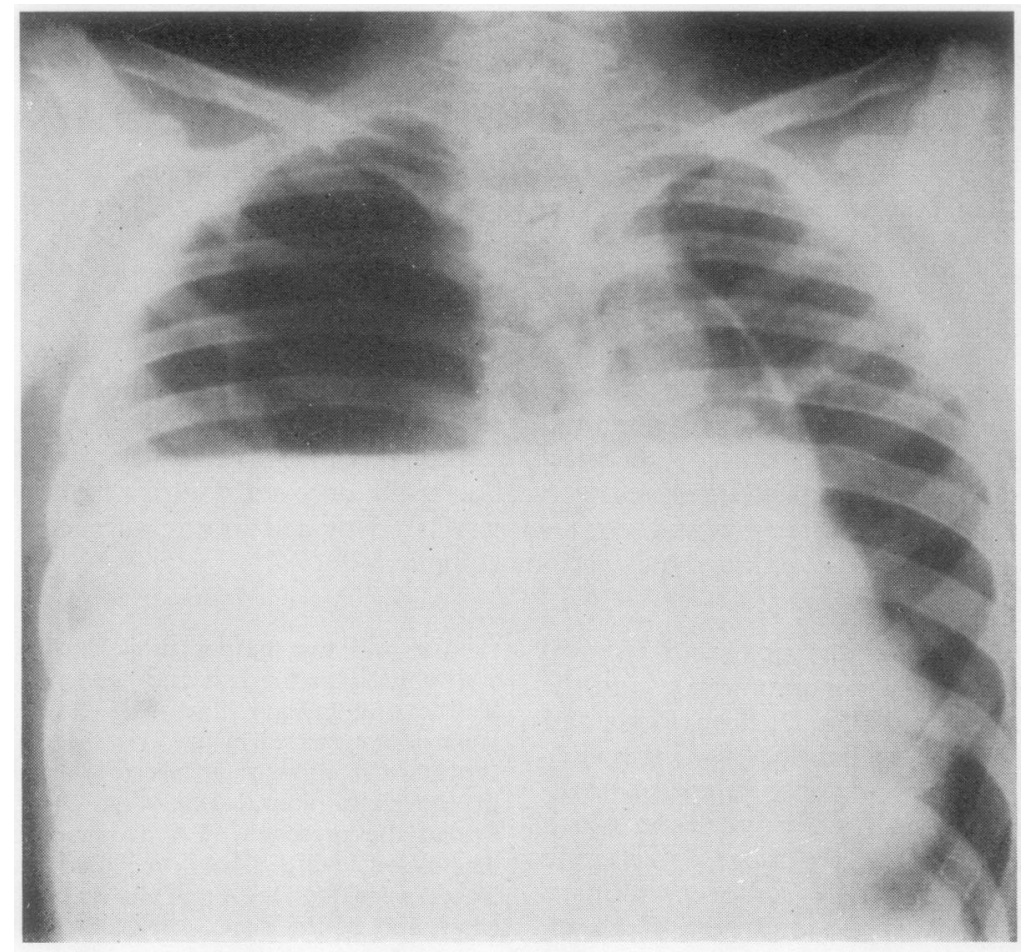

(a)

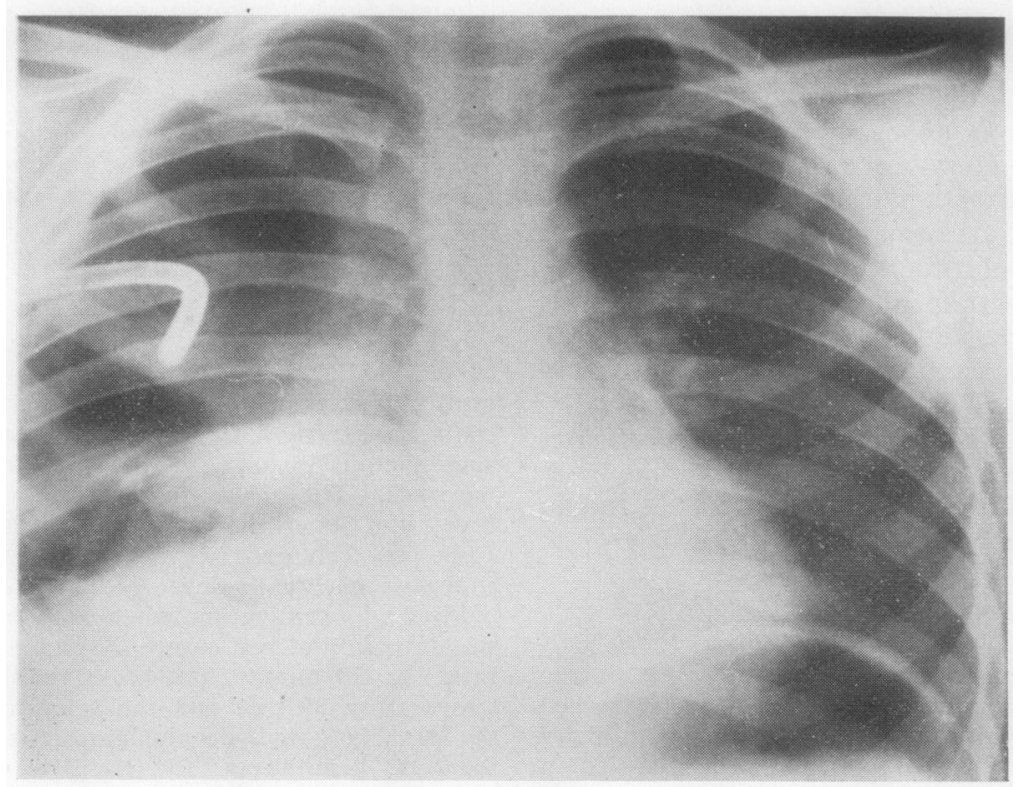

(b)

Legend to Fig. 1 (a) and (b) on page 549. 


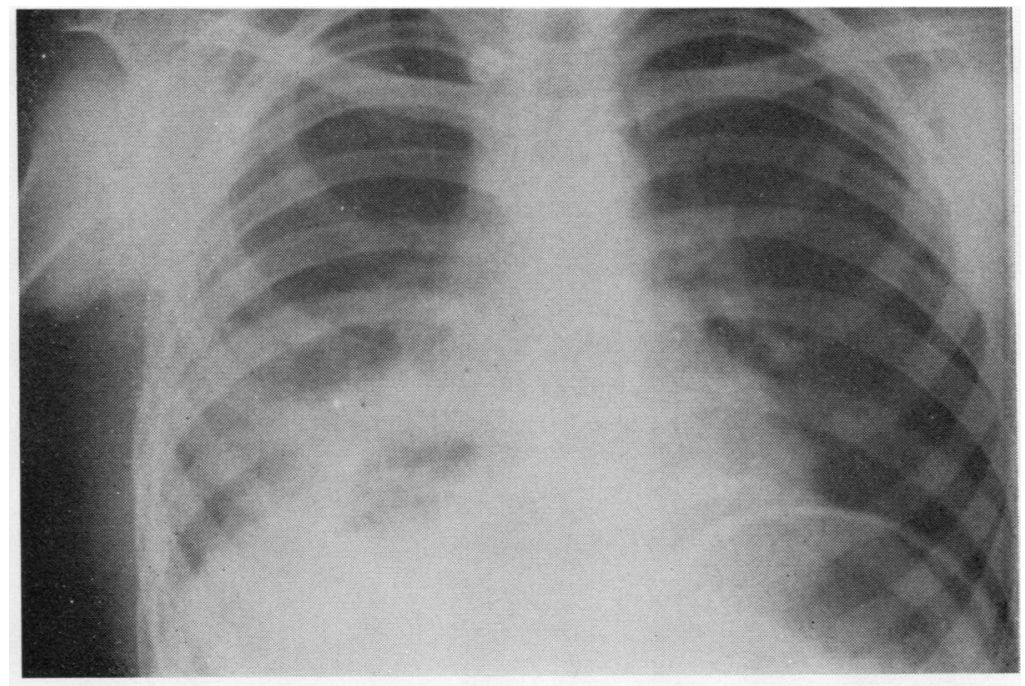

(c)

FIG. 1. Case 1. (a) Hydropneumothorax with marked mediastinal shift. (b) After a water seal drainage and suction, double wall rounded shadow is seen at the base of the right lung. (c) Post-operative film. The lung is fully expanded. The shadow at the right base is a residual pleural reaction.

the right pleural cavity. A round shadow was visualized in the collapsed right lobe and a second one was seen in the upper zone of the left lung (Fig. 2a). The W.B.C. was $10,200 / \mathrm{cu}$. $\mathrm{mm}$. and there were $8 \%$ eosinophils. The Casoni skin test and the Weinberg complement-fixation test were negative. Serosanguineous fluid came through a water seal drainage, but this last procedure did not succeed in relieving the collapse (Fig. 2b). A thoracotomy was performed and a ruptured hydatid cyst in the right lower lobe was found. Lobectomy was done and another cyst was enucleated from the right upper lobe, after which recovery was uneventful and the lung fully expanded (Fig. 2c).

CASE 3 A 40-year-old woman experienced a sharp, stabbing, tearing pain in the left hemithorax following a simple sneeze. Her past history was not significant except for removal of a hydatid cyst from the kidney a few years previously.

Examination revealed a seriously ill woman who was orthopnoeic with a respiratory rate of $35 /$ minute and a pulse rate of $110 /$ minute. The temperature was $38^{\circ} \mathrm{C}$., the trachea was shifted to the right, the apex beat was felt in the right hemithorax, and the heart sounds were audible in the right chest. The left chest was hyper-resonant, the breath sounds were absent, and the vocal resonance was diminished. A chest radiograph showed complete collapse of the left lung, with shift of the upper and lower mediastinum toward the opposite side (Fig. 3a). The W.B.C. count was
$9,000 / \mathrm{cu}$. mm. and the eosinophils were 5-11\%. The Casoni skin test was positive. Aspiration revealed the high tension quality of the pneumothorax. Continuous air leak and failure of expansion of the lung in spite of continuous suction confirmed the presence of a fair sized broncho-pleural fistula. When thoracotomy was carried out, a ruptured hydatid cyst was removed from the lingula, and the fistula was closed. Recovery was satisfactory and the lung expanded fully (Fig. 3b).

CASE 4 A 33-year-old woman was seized suddenly with severe left-sided chest pain. The pain was associated with shortness of breath, cough, and expectoration of a large amount of salty fluid. She was referred to a county hospital where a chest radiograph was taken, after which she was referred to our thoracic surgical unit for management.

Examination revealed a well-built woman who was short of breath and perspiring. The pulse rate was $110 /$ minute. There was hyperresonance in the lower part of the left hemithorax, and the cardiac dullness shifted to the right hemithorax. The chest radiograph on admission showed a large air space in the lower part of the left chest with what seemed to be a waterlily appearance. Moderate shift of the lower mediastinum to the opposite side was present. There were linear opacities at the upper border of the air space reaching the chest wall (Fig. $4 a, b$ ).

Under-water seal drainage was placed in the midaxillary line, where there was a considerable air leak and some fluid came out. This procedure was of no 


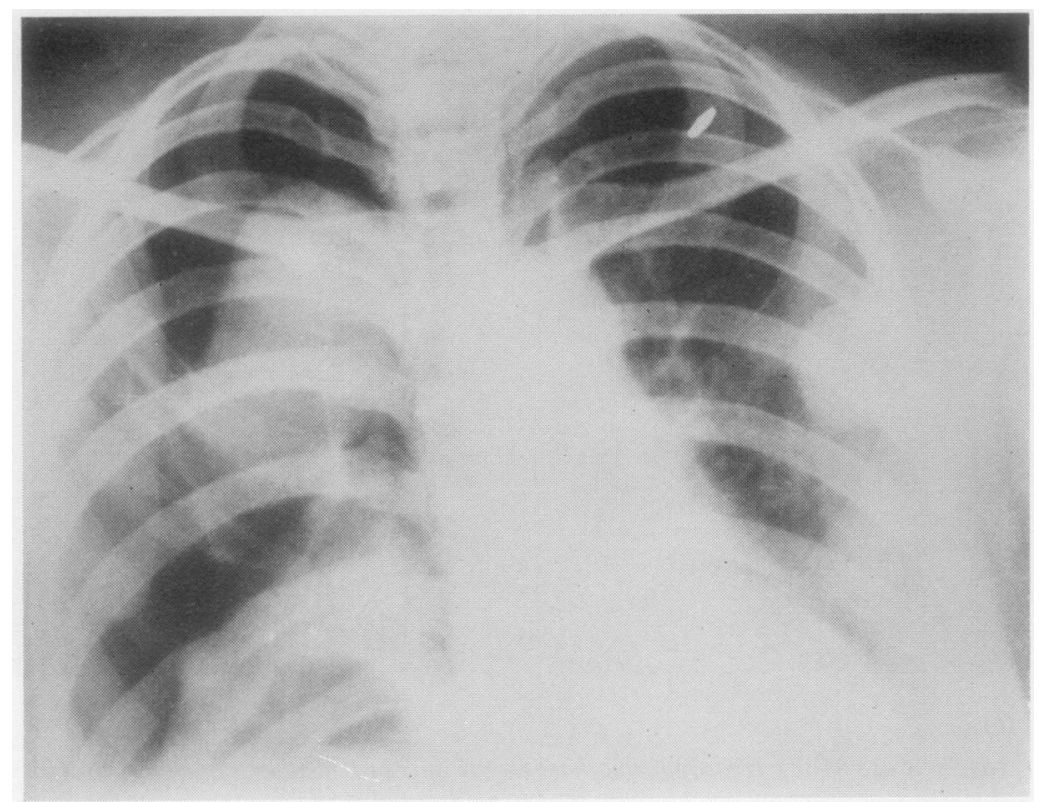

(a)

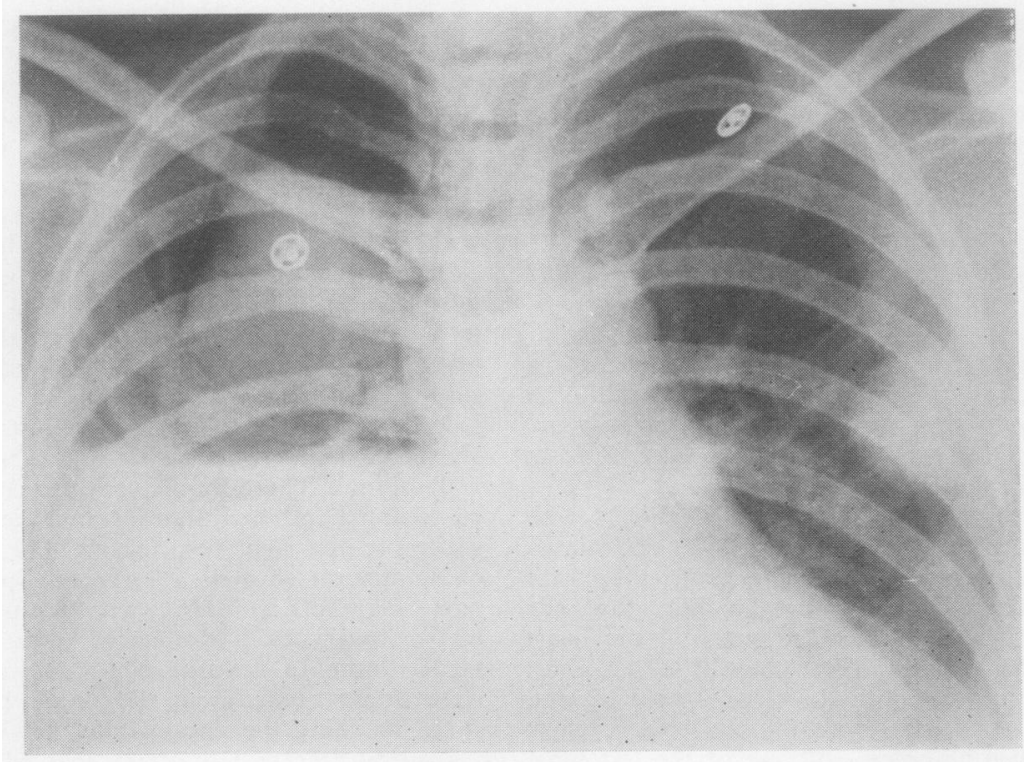

(b)

Legend to Fig. 2 (a) and (b) on page 551. 


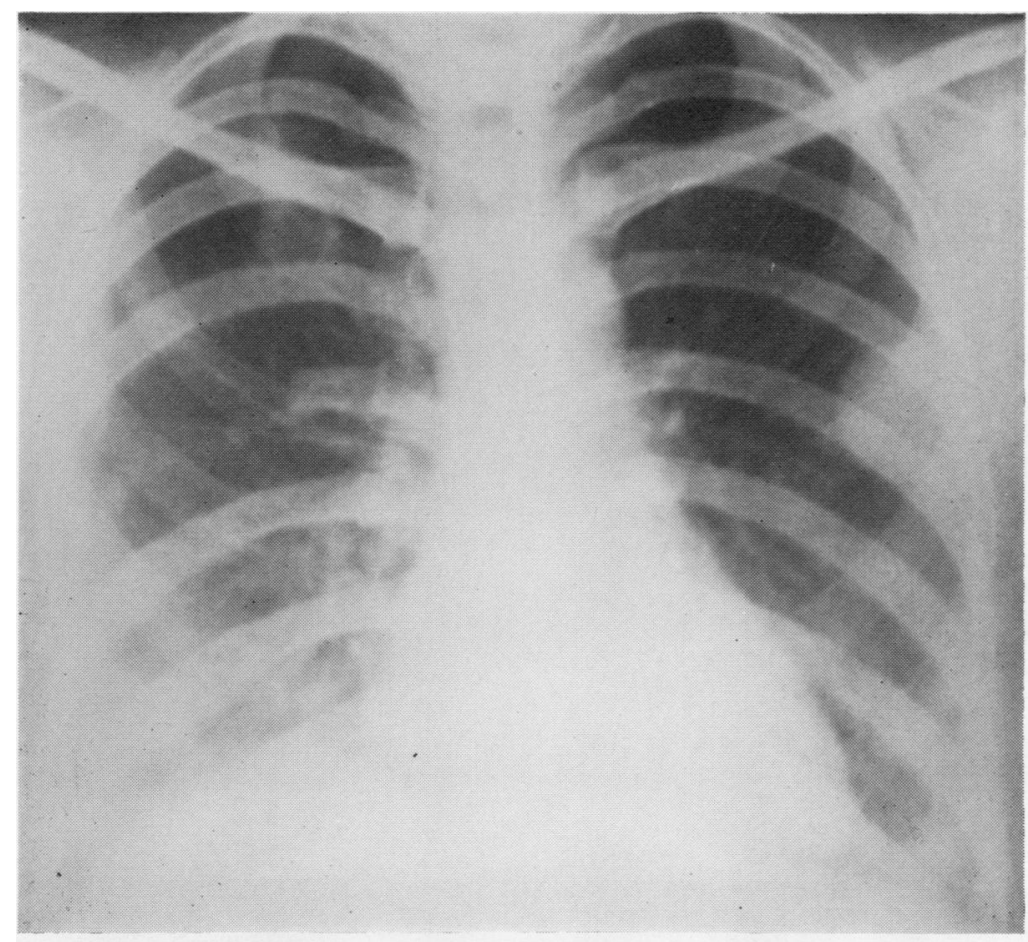

(c)

FIG. 2. Case 2. (a) Right pneumothorax with a collapsed lung, a rounded shadow in the right upper lobe and a second one in the left mid-zone area. (b) After aspiration, an air-fluid level is seen. No lung expansion was achieved. (c) Postoperative film. Complete correction with full expansion: the rounded shadow in the left lung is still seen.

help in expanding the lung. Examination of the fluid was negative for scolices but the Casoni skin test was positive.

Thoracotomy was performed, when a large ruptured hydatid cyst was found occupying the anterolateral aspect of the left lower lobe, and a $0 \cdot 3-\mathrm{cm}$. tear was found in the pleura. The whole upper lobe was adherent to the chest wall. The cyst was removed, the leaking bronchus was closed, the space was obliterated, and the chest was closed with basal and apical drainage. The post-operative course was uneventful.

CASE 5 An 18-year-old girl complained of severe pleuritic pain of seven days' duration. The pain was of gradual onset and was associated with cough, expectoration of whitish sputum, and a continuous low-grade fever. The patient gave a history of haemoptysis six years previously, for which she had been admitted to a sanatorium.

On physical examination the patient was actually ill and in pain, the pulse rate was $120 /$ minute, the temperature was $38.5^{\circ} \mathrm{C}$., and the respiratory rate was
$40 /$ minute. The trachea was central on admission. The left upper chest was slightly more resonant than the right, but there were signs of a small effusion in the left axilla. The lower border of the liver was felt $3 \mathrm{~cm}$. below the right costal margin and was not tender. The spleen was felt $5 \mathrm{~cm}$. below the costal margin: the uterus was the size of a 32 weeks' pregnancy. The admission chest radiograph showed a small pneumothorax on the left side and a cavitylike appearance in the collapsed lung with pleural thickening and effusion (Fig. 5a). The W.B.C. count ranged from 15,000 to $11,000 / \mathrm{cu}$. $\mathrm{mm}$. The polymorphs ranged from 90 to $73 \%$ and the eosinophils were within normal limits. The Casoni skin test was positive.

Two days after admission to hospital the patient developed severe respiratory distress. The trachea shifted to the opposite side and signs of tension pneumothorax were evident. A further chest radiograph showed obvious mediastinal shift, a collapsed lung with an empty cavity in it, and a fluid level at the lower part of the pleural cavity (Fig. 5b). A water-seal drainage was placed in the second anterior space. 


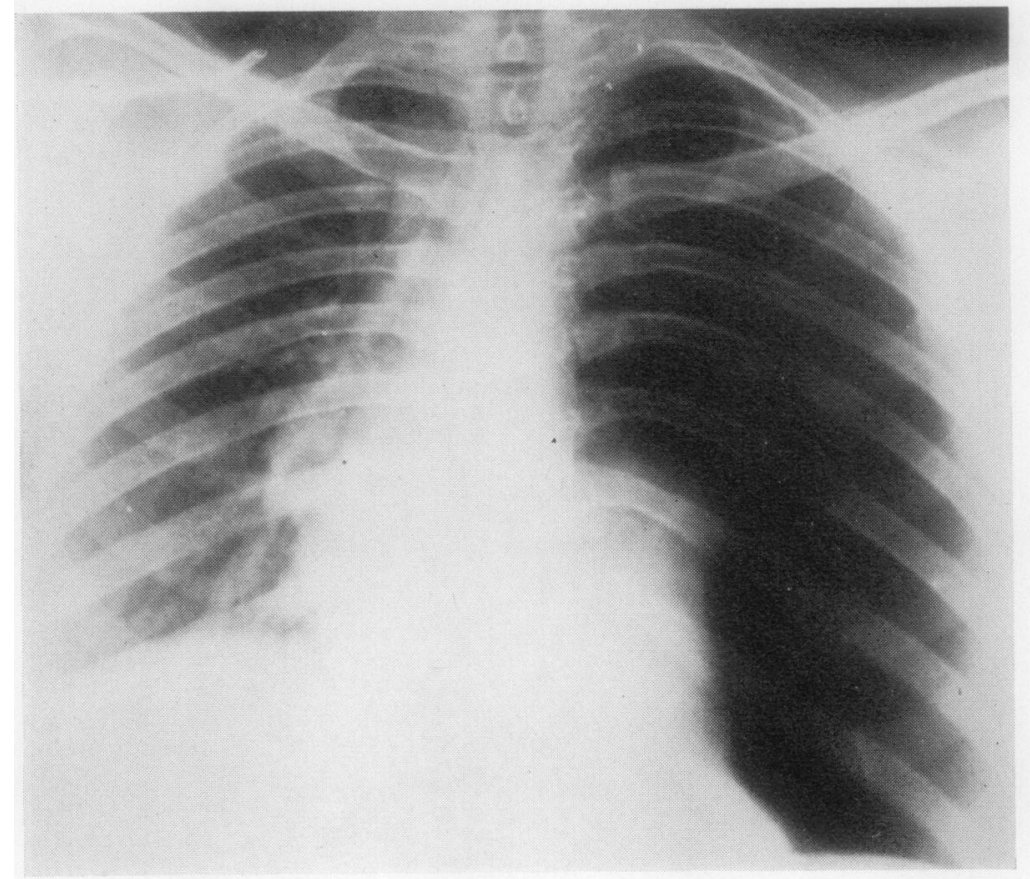

(a)

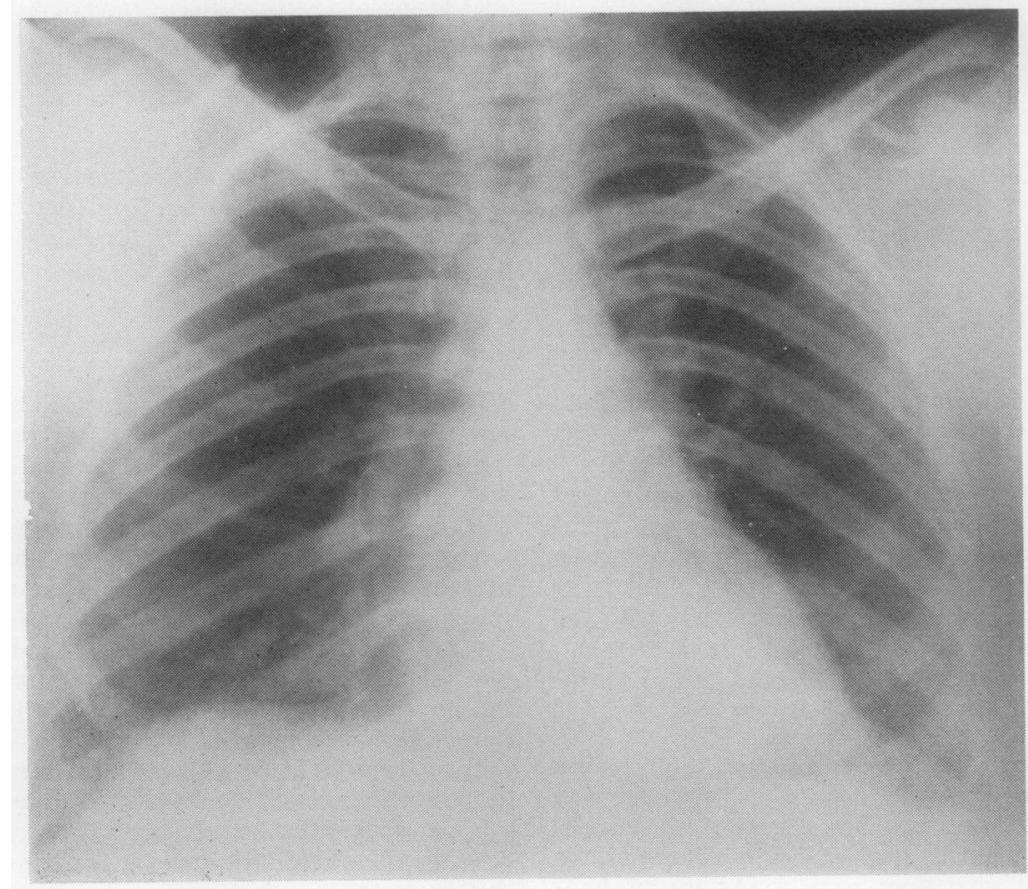

(b)

FIG. 3. Case 3. (a) Massive left pneumothorax with severe mediastinal shift and complete atelectasis of the left lung. (b) Post-operative film. Full expansion with a normal chest radiograph. 

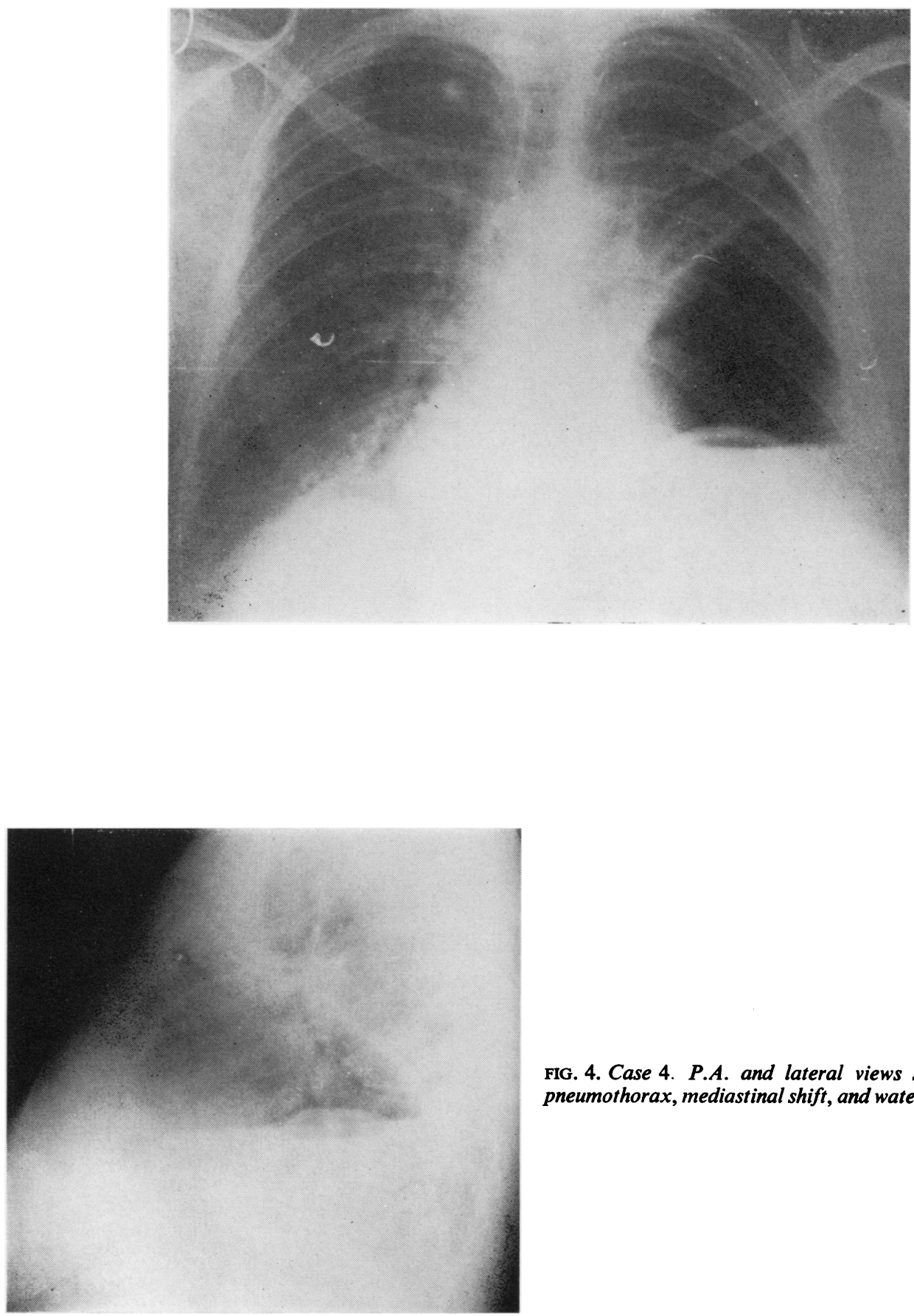

FIG. 4. Case 4. P.A. and lateral views showing selective pneumothorax, mediastinal shift, and water-lily appearance. 


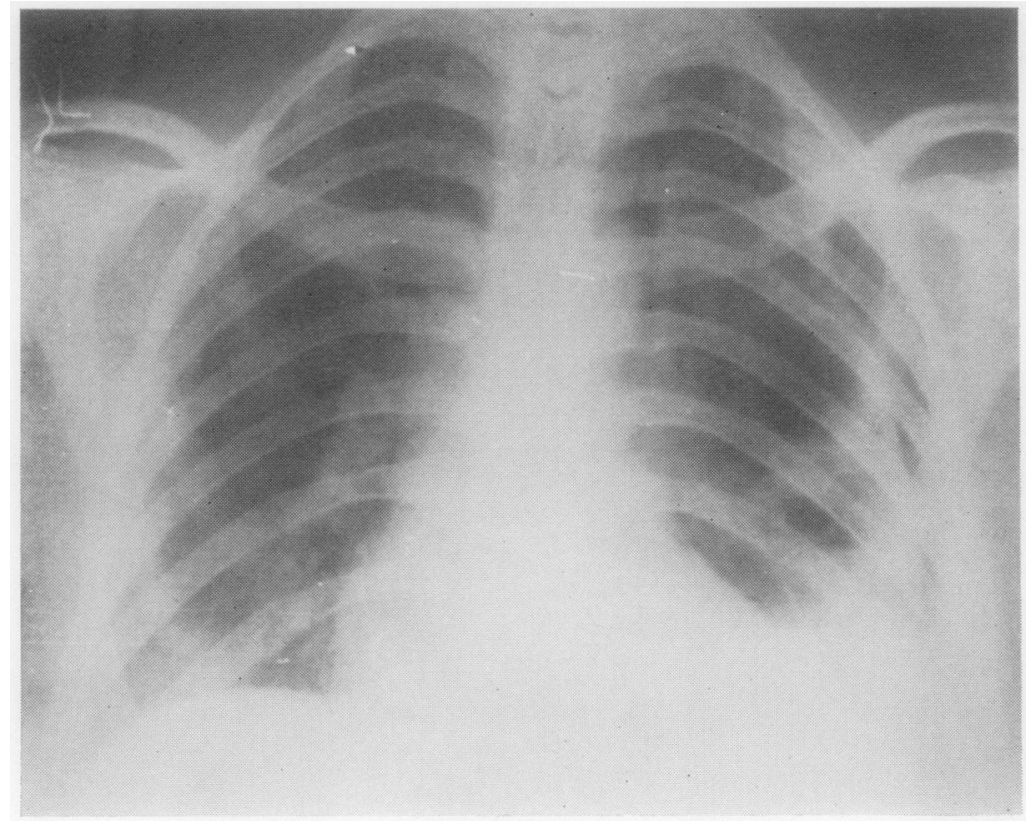

(a)

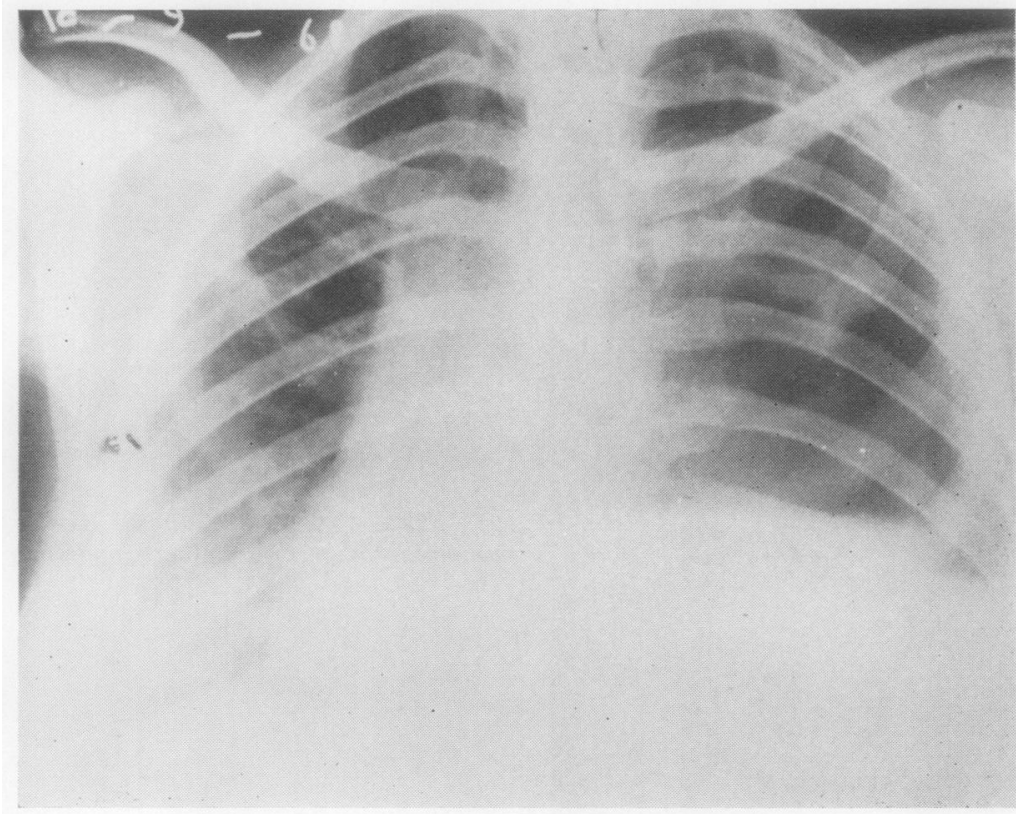

(b)

FIG. 5. Case 5. (a) Small pneumothorax and pleural thickening and effusion in the collapsed lung. (b) Tension pneumothorax two days after admission. 
Unmistakable hydatid material was recovered from the fluid aspirated posteriorly. The patient gave birth to a normal infant prematurely, and she is still in hospital under conservative measures for the infected cavity.

\section{DISCUSSION}

Hydatid cyst is a continuously growing parasite. Its expansion is usually resisted by the tissue and organ of the host, though this reaction of the lung parenchyma is minimal and poor as compared with that of other organs (Cleland, 1963). Pulmonary echinococcal cysts may reach a huge size during growth, the eroding blood vessel often giving rise to haemoptysis. The bronchial passages could be eroded as well and air escape around or into the cyst, this accounting for the various radiological features in this disease. The eroded bronchus does not function as a fistula to start with, due to the pressure of the tense cyst against the fistula opening. Progressive enlargement will lead to its rupture either into the bronchial tree or into the pleura. Intrapleural rupture more frequently presents as pneumo- or hydropneumothorax. Occasionally such an accident may present in one of the allergic manifestations such as urticaria, asthma, or dramatic anaphylactic shock.

The pneumothorax may be tension in type, as in the above cases, where it is quite easy to visualize the tension or the valvular mechanism. The collapsed or crumpled wall of a ruptured cyst simply acts as a curtain or check valve, admitting air into the pleura during inspiration, then falling back and closing the opening or outlet during expiration, and thus allowing air to enter but not to escape from the pleural cavity (Fig. 6). A positive pressure will continuously build up inside the pleural cavity which will ultimately shift the mediastinal structures to the opposite side, interfering with the haemodynamics of the respiratory and cardiovascular systems. These changes are responsible for the clinical picture usually seen in tension pneumothorax which may be fatal if the tension is not relieved.

All the five cases reported here illustrate the tension quality of the pneumothorax both clinically and radiologically. Because of the rather dramatic clinical picture, manometric measurement was not attempted. A sudden rise in the intrapulmonary pressure is the usual precipitating factor in rupture of the cyst: the cause could be a trivial one, such as coughing or sneezing, though sometimes it may follow an increase in intraabdominal pressure as in pregnancy or ascites. Rupture may occur spontaneously without any predisposing factor. As a result, secondary hydatidosis of the pleura may result from seeding of the scolices into the pleural cavity (Deve, 1925; Tomalino, 1959). Pleural infection and empyema is frequently met with, as in cases 1,2 , and 5 . It is quite possible that the infection reaches the space via a trans-bronchial spread: infection and empyema are always an additive insult to the patient and increase the seriousness of the illness.

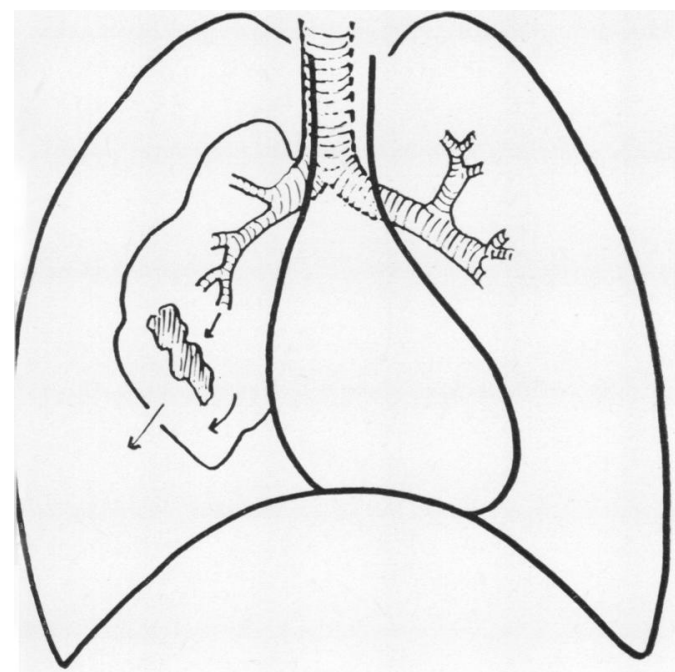

(a)

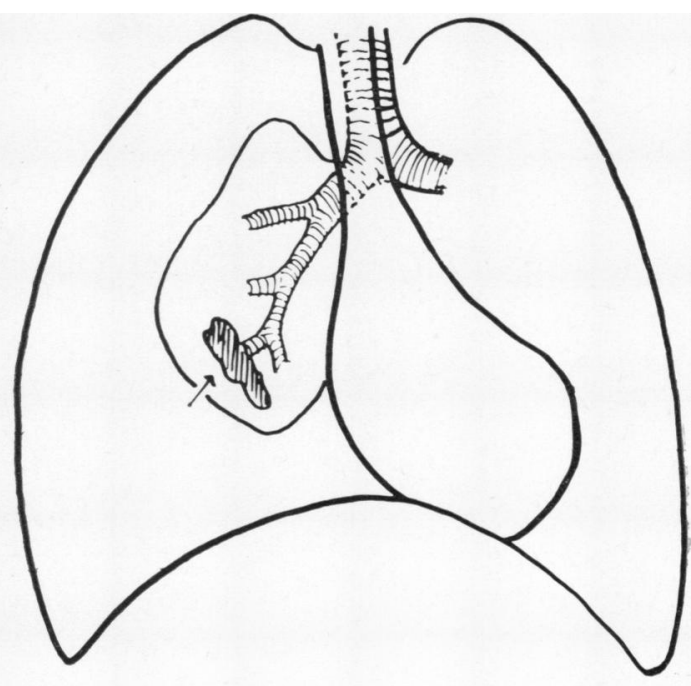

(b)

FIG. 6. Mechanism of echinococcal tension pneumothorax: (a) during inspiration; (b) during expiration. 
Since pulmonary hydatid cysts are liable to a number of serious complications (Bakir, 1967), we should not leave such cysts waiting for spontaneous evacuation or natural cure. Coughing up the cyst is usually associated with complications (Brown, 1958 ; Cleland, 1963), and in our opinion complete evacuation with a satisfactory natural cure is exceptional.

Since the natural course in any single case cannot be foreseen, we strongly advocate surgical removal of any pulmonary hydatid cyst or suspected cyst as soon as it is discovered. The recent advances made in thoracic surgery and anaesthesia are an additional justification for a surgical approach. Earlier operation is easier and less devastating and a cure may be achieved by simple enucleation of the cyst. Conservative measures such as needling and water-seal drainage with or without suction have only succeeded in alleviating the distressing emergency symptoms but never in closing the fistula and expanding the lung: we believe that thoracotomy is mandatory after relieving the tension by these measures.

Conventional diagnostic measures such as the Casoni skin test, the Weinberg complement fixation test, and an eosinophil count are not very reliable when the cyst has ruptured or is infected or calcified. Some features made us suspect an echinococcal nature or aetiology in our cases, such as the radiological shadow of a cyst wall in case 1 (Fig. $1 \mathrm{~b}$ ), the multiple rounded shadows in case 2 (Fig. 2a), a previous history of a hydatid cyst removed from a kidney in case 3 , the expec- toration of salty fluid and a water-lily appearance in case 4 , and an empty cavity with aspiration hydatid material in case 5 . Other helpful diag nostic points are urticaria, asthma, or anaphylaxes as the allergic manifestation of a ruptured cyse, while scolices in the pleural aspirate are pathe gnomonic. A tension pneumothorax with persisting broncho-pleural fistula in an endemic area of the

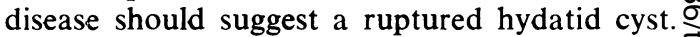

We should like to thank Dr. Kwakab M. Hickmat for Figure 6 illustrating the mechanism of tensigh pneumothorax.

\section{REFERENCES}

Agustoni, C. B., Taboada F. F., Berinstein, E., and Chorne, I. (1960) 767 Espontaneo hidatideo. Sem. méd. (B. Aires). Fio Neumo thorax, 116.

Bakir, F. (1967). Serious complications of hydatid cyst of the lung. Amer. Rev. resp. Dis., 96, 483 .

Brown, C. J. O. (1958). Surgical pathology of hydatid cysts of the lur Postgrad. med. J., 34, 195.

Cleland, W. P. (1963). Pulmonary hydatid disease. In Chest Diseas Vol. 2, p. 386. Ed. Perry, K. M. A., and Sellors, T. H. Butte worths, London.

DuBose, H. M., Price, H. J., and Guilfoil, P. H. (1953). Spontaneouto pneumothorax: medical and surgical management. Analysis of 75 patients. New Engl. J. Med., 248, 752.

Kelly, T. D., and Izzi, N. (1959). Pulmonary hydatid disease in Irag with a review of history and life history of the parasite. $J$. Fod Med. Baghdad, n.s., 1, 115

Mills, M., and Baisch, B. F. (1965). Spontaneous pneumothorax series of $\mathbf{4 0 0}$ cases. Ann. thorac. Surg., 1, 286.

Perry, K. M. A. (1939). On spontaneous pneumothorax. Quart. $\bar{Q}$ Med., n.s., $8,1$.

Sala de Pablo, J., and San Joaquin, R. Araiz (1954). Neumotor hidatidico. Cirurg. Ginec. Urol., 7, 171.

Sinclair, J. D. (1955). Spontaneous pneumothorax. A review of sevent cases. N.Z. med. J., 54, 51 .

Tomalino, D. (1959). Pleural complications of hydatidosis, pleuren hydatid accident, secondary pleural hydatidosis (study of 47 personal observations). Tòrai, , 8, 73.

Waddle, N. (1950). Pulmonary hydatid disease. A review of 478 cass reported in the Louis Barnett Hydatid Registery of the Royal Australasian College of Surgeons. Aust. N.Z. J. Surg., 19, 273. 